\title{
Laser-Induced Oxidation of Cholesterol Observed During MALDI-TOF Mass Spectrometry
}

\author{
Kevin M. McAvey, Bing Guan, Chanel A. Fortier, Matthew A. Tarr, Richard B. Cole \\ Department of Chemistry, University of New Orleans, 2000 Lakeshore Dr., New Orleans, LA, 70148, USA
}

\begin{abstract}
Conditions for the detection of three odd-electron cholesterol oxidation peaks were determined and these peaks were shown to be artifacts of the matrix-assisted laser desorption time of flight (MALDI-TOF) process. Matrix choice, solvent, laser intensity and cholesterol concentration were systematically varied to characterize the conditions leading to the highest signals of the radical cation peaks, and it was found that initial cholesterol solution concentration and resultant density of solid cholesterol on the MALDI target were important parameters in determining signal intensities. It is proposed that hydroxyl radicals, generated as a result of laser irradiation of the employed 2, 5-dihydroxybenzoic acid (DHB) matrix, initiate cholesterol oxidation on the MALDI target. An attempt to induce the odd-electron oxidation peaks by means of adding an oxidizing agent succeeded using an acetonitrile solution of DHB, cholesterol, and cumene hydroperoxide. Moreover, addition of free radical scavengers reduced the abundances of some oxidation products under certain conditions. These results are consistent with the mechanism of oxidation proposed herein involving laser-induced hydroxyl radical production followed by attack on neutral cholesterol. Hydroxyl radical production upon irradiation of dithranol matrix may also be responsible for generation of the same radical peaks observed from cholesterol in dithranol by an analogous mechanism.
\end{abstract}

Key words: Cholesterol, Cholesterol oxidation, Oxidation mechanism, Radical ions, MALDI

\section{Introduction}

$\mathrm{M}$ ALDI-MS is a valuable tool for the study of oxidation products from biological transformations. Cholesterol is a highly studied biological compound due to its structural importance in lipid membranes and the damaging effects caused by its oxidation [1-5]. Certain cholesterol oxidation products (COPs) have been shown to be carcinogenic and

Electronic supplementary material The online version of this article (doi:10.1007/s13361-011-0074-3) contains supplementary material, which is available to authorized users.

Correspondence to: Richard B. Cole; e-mail: rcole@uno.edu mutagenic [6]. Owing principally to the presence of a lone double bond (Scheme 1), cholesterol is susceptible to oxidation by means of enzymes, autoxidation, and photooxidation [7]. The high propensity for cholesterol to undergo oxidation has led some to believe that complete removal of COP artifacts may be impossible [7].

In a previous paper, cholesterol was shown to be oxidized by the Fenton reagents and the products were subsequently characterized by MALDI-TOF/TOF-MS [8]. During the course of that study, low intensity even-mass peaks representing radical cations sporadically appeared in mass spectra. There was no obvious reason for these presumably short-lived open-shell cations to be present on the target. Cholesterol oxidation kinetics have been studied [9], and it 


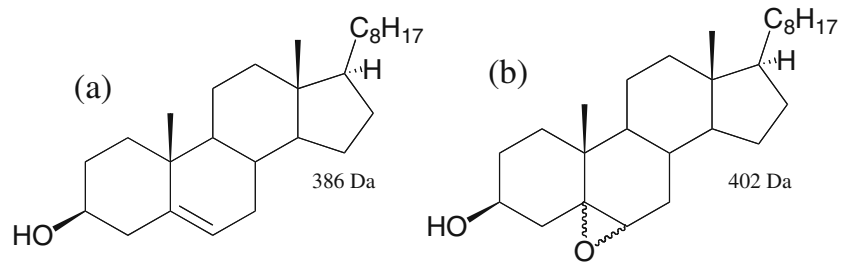

(c)

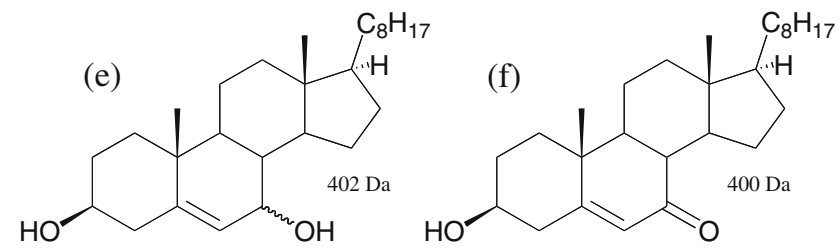

Scheme 1. Structures of cholesterol and known cholesterol

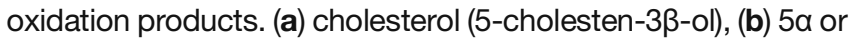
$5 \beta$-epoxycholesterol, (c) $7 \alpha$ - or $7 \beta$-hydroperoxycholesterol, (d) $5 \alpha$ - or $5 \beta$-hydroperoxycholesterol, (e) $7 \alpha$ - or $7 \beta$-hydroxycholesterol, (f) 7-ketocholesterol (5-cholesten-3 $\beta$-ol-7-one)

is believed that any oxidation intermediates that correspond to radicals should have been transformed into the ultimate oxidation products rather rapidly.

The oxygen-mediated and radical-mediated oxidation pathways of cholesterol have been studied extensively [10-12]. Smith [12] has shown that oxidation of cholesterol via the excited singlet state molecular oxygen pathway implicates $3 \beta$-hydroxy-5 $\alpha$-cholest-6-ene-5-hydroperoxide and $7 \alpha$-hydroperoxide, whereas oxidation via the free radical mechanism involves 7-peroxy radicals or 7hydroperoxides.

There are over 70 known oxidation products of cholesterol; this fact serves as evidence of the complexity of cholesterol oxidation [12]. Many oxidation products of cholesterol have been studied by GC-MS [13, 14], EI-MS [15], SIMS [16], and HPLC [17, 18]. Each technique leaves its own unique artifacts and has its own drawbacks [7]. The chromatographic techniques generally have poor resolution when separating individual oxidation products [3]. Cholesterol is generally described as a poor candidate for analysis by ES-MS and MALDI-TOF due to poor ion yields [19]. Normally, cholesterol is subjected to a derivatization reaction such as use of Girard P hydrazine [20] or the addition of an acetyl group [15] to increase ion yields in GCMS. In the current MALDI-TOF study, cholesterol was left unaltered which led to relatively low intensity peaks. However, improvements in MALDI-TOF sensitivity, mass accuracy and resolution have made it possible to detect and unambiguously identify cholesterol, its oxidation products, and artifacts.
In this paper, the conditions leading to the detection of even-mass radical cation peaks are investigated. Mechanisms for the formation of radical cholesterol species are proposed. The effects of the addition of radical scavengers and a radical initiator are also discussed.

\section{Experimental}

\section{Materials}

Cholesterol was purchased from MC\&B (Norwood, OH, USA) and Aldrich (St. Louis, MO, USA). HPLC grade chloroform was purchased from EMD (Darmstadt, Germany). Methanol was purchased from Sigma-Aldrich in HPLC grade. Acetonitrile was purchased from Sigma-Aldrich in greater than $99.9 \%$ purity. Deionized distilled water was obtained from a Milli-Q water system (Billerica, MA, USA). 2,5-Dihydroxybenzoic acid (DHB), dithranol, and meso-tetrakis porphyrin (MTP) matrixes were purchased from Aldrich. Tertiary butylhydroquinone (TBHQ), $\alpha$-tocopherol, cumene hydroperoxide, 5-cholestene$3 \beta, 7 \beta$-diol (greater than $98 \%$ purity), 5 -cholesten-3 $\beta$-ol-7-one (greater than $90 \%$ purity), and cholesterol $5 \beta, 6 \beta$-epoxide (greater than $98 \%$ purity) were all purchased from Aldrich.

\section{Sample Preparation}

Cholesterol was dissolved in three different solvent mixtures (vol:vol): $\mathrm{CH}_{3} \mathrm{OH}: \mathrm{H}_{2} \mathrm{O}$ (10:1), $\mathrm{CHCl}_{3}: \mathrm{CH}_{3} \mathrm{OH}$ (1:1), and acetonitrile. Three different matrixes were tested: 2,5-dihydroxybenzoic acid (DHB) in a concentration of $10 \mathrm{mg} / \mathrm{mL}$, dithranol at $10 \mathrm{mg} /$ $\mathrm{mL}$ and meso-tetrakis porphyrin (MTP) [21] at $10 \mathrm{mg} / \mathrm{mL}$. An aliquot of $10 \mu \mathrm{L}$ of sample solution was mixed with $40 \mu \mathrm{L}$ of matrix solution in a small microcentrifuge tube (Eppendorf, Westbury, New York). MALDI samples were prepared using the 'dried droplet' method [22]. For the radical scavenger samples, a $3 \mu \mathrm{L}$ aliquot of concentrated $\alpha$-tocopherol was added to $50 \mu \mathrm{L}$ solutions containing $40 \mu \mathrm{L}$ of $10 \mathrm{mg} / \mathrm{mL}$ DHB in $1: 1 \mathrm{CHCl}_{3}: \mathrm{CH}_{3} \mathrm{OH}$ and $10 \mu \mathrm{L}$ of approximately $0.04 \mathrm{mg} /$ $\mathrm{mL}$ cholesterol in 1:1 $\mathrm{CHCl}_{3}: \mathrm{CH}_{3} \mathrm{OH}$ (1000:1 wt:wt); reaction time was $5 \mathrm{~min}$. This was repeated for the other two solutions (10:1 $\mathrm{CH}_{3} \mathrm{OH}: \mathrm{H}_{2} \mathrm{O}$ and acetonitrile). TBHQ was dissolved in each solvent system at a concentration of approximately $45 \mu \mathrm{M}$ and a $0.5 \mu \mathrm{L}$ aliquot was added to each different solvent/matrix solution and allowed to react for $5 \mathrm{~min}$. For the radical initiator studies, a $1 \mu \mathrm{L}$ aliquot of $80 \%$ cumene hydroperoxide was added to each 1000:1 wt:wt matrix-to-analyte solution. After the addition of the radical scavenger or initiator, the solution was mixed for 30 seconds and $0.5 \mu \mathrm{L}$ of the final solution was spotted onto the MALDI plate.

\section{Instrumentation}

Cholesterol oxidation products were studied using a matrixassisted laser desorption ionization time of flight/time of flight mass spectrometer (AB SCIEX, Concord, Ontario, Canada, 4800 MALDI-TOF/TOF) equipped with a $355-\mathrm{nm}$ 
Nd:YAG laser with a $200-\mathrm{Hz}$ firing rate. Manual mode was used for all measurements. MALDI mass spectra were acquired in the positive ion reflectron mode. MALDI mass spectra were calibrated using known matrix and cholesterol peaks (internal calibration). Electrospray (ES) mass spectra were obtained on an AB SCIEX 3200 Qtrap. The flow rate was set at $5 \mu \mathrm{L} / \mathrm{min}$. All acquisitions were made in the positive ion mode using single quadrupole mass analysis.

\section{Results and Discussion}

Figure 1a shows the MALDI-TOF mass spectrum of cholesterol from a 1:1 solution of $\mathrm{MeOH}: \mathrm{CHCl}_{3}$ with $\mathrm{DHB}$ as the matrix. In addition to $\mathrm{MH}^{+}$at $m / z 387$, rather intense peaks can be seen at $m / z 384,400$, and 402 . By the nitrogen rule, it can be deduced that these latter peaks represent radical cations arising from the cholesterol sample. It seems reasonable that these observed radical products were, in fact, not solution-based oxidation products, because open-shell species are not expected to be long-lived. Rather, we propose that they are products of laser irradiation that have been formed during the MALDI process.

\section{Matrix Influence}

It is generally accepted that matrix-solvent composition and sample preparation greatly influence the quality of MALDI mass spectra [23]. MTP, dithranol and DHB were tested in all three solvent systems to see if the matrix had any effect on the formation of radical cation peaks of cholesterol. MTP did not produce readily detectable cholesterol peaks, nor did any radical cation species related to cholesterol appear in the mass spectra. DHB has previously been shown to be a suitable matrix for the analysis of cholesterol by MALDI-TOF [24]; even though under certain conditions dithranol did produce cholesterol peaks, including radical species, $\mathrm{MH}^{+}$peaks for cholesterol were more intense from DHB, hence, the latter was chosen as the most suitable matrix for the analysis of cholesterol.

\section{Solvent Choice}

Figure $1 \mathrm{~b}$ shows the MALDI-TOF mass spectrum of cholesterol that was prepared in acetonitrile with DHB as the matrix. In acetonitrile the $\left[\mathrm{M}+\mathrm{H}-\mathrm{H}_{2} \mathrm{O}\right]^{+}$peak at $\mathrm{m} / \mathrm{z}$ 369 was the dominant peak. Figure 2a shows cholesterol that was prepared in 10:1 $\mathrm{CH}_{3} \mathrm{OH}: \mathrm{H}_{2} \mathrm{O}$ with $\mathrm{DHB}$ as the matrix. In this latter solvent, as well as in acetonitrile, the $[\mathrm{M}+\mathrm{H}]^{+}$ peak at $\mathrm{m} / \mathrm{z} 387$ was almost never seen. In 1:1 $\mathrm{CHCl}_{3}$ : $\mathrm{CH}_{3} \mathrm{OH}$ solvent (Figures 1a and 2c, d), the $[\mathrm{M}+\mathrm{H}]^{+}$peak was observed on a consistent basis. Another general difference distinguishing $\mathrm{CHCl}_{3}: \mathrm{CH}_{3} \mathrm{OH}$ from $\mathrm{CH}_{3} \mathrm{OH}: \mathrm{H}_{2} \mathrm{O}$ or acetonitrile is the latter two solvents propensity to form $\mathrm{m} / \mathrm{z}$ 401, which will be discussed later. In all three solvent systems, using DHB as the matrix, even-mass radical cation peaks would sporadically appear.

\section{Laser Intensity}

The laser intensity was varied from 3500 to 5500 (arbitrary units); a value of 3900 corresponds to a level just above the threshold to desorb ionic products. Figure $2 \mathrm{a}$ shows the MALDI-TOF mass spectrum of cholesterol with DHB as the matrix in $\mathrm{MeOH}: \mathrm{H}_{2} \mathrm{O}$ acquired at a laser intensity of 4150 . Figure $2 \mathrm{~b}$ shows the spectrum from an identically prepared target acquired at a laser intensity of 5050. Figure $2 \mathrm{c}$ and d show the spectra of cholesterol prepared in $\mathrm{CHCl}_{3}: \mathrm{CH}_{3} \mathrm{OH}$ at laser intensities of 4150 and 5050, respectively. While laser intensities below 4100 showed very little propensity to induce cholesterol ionization, higher intensities tended to increase the probability of detecting peaks at $\mathrm{m} / \mathrm{z} 400$ and 402 , but at the cost of extremely high chemical noise, i.e., peaks were appearing at every $m / z$ value in this range. At higher laser intensities, the radical cation peaks appeared sporadically, but were not detectable on a consistent basis with the exception of $\mathrm{m} / \mathrm{z} 384$.

\section{Identification and Origin of Oxidation Products}

Peaks can be seen at $m / z$ 384, 400, and 402 in Figure 1a. It is highly unlikely that these open-shell cations are stable while on the target, which leads to the alternative explanation that the peaks are most likely the result of laser-induced reactions. Blanks were run and it was demonstrated that the peaks do not arise from the matrix or solvent. Employing an internal calibration using known DHB and cholesterol fragmentation peaks, the accurate masses of the radical cation peaks were determined. The peak at $\mathrm{m} / \mathrm{z} 402.3484$ corresponds to $\left[\mathrm{MH}+\mathrm{OH}-\mathrm{H}_{2}\right]^{+\cdot}$ (2.1 ppm error) while the peak at $\mathrm{m} / \mathrm{z} 400.3323$ corresponds to $\left[\mathrm{MH}+\mathrm{OH}-2 \mathrm{H}_{2}\right]^{+}$ (3.2 ppm error) and the peak at $\mathrm{m} / \mathrm{z} 384.3391$ corresponds to $\left[\mathrm{MH}+\mathrm{OH}-\mathrm{H}_{2}-\mathrm{H}_{2} \mathrm{O}\right]^{+\cdot}$ (1.1 ppm error). Previous work into the cholesterol oxidation pathway by Smith [12] and Uemi et al. [11] led to the deduction that solution oxidation products contain hydroperoxide structures (Scheme 1c and d). However, there is no source of molecular oxygen or peroxide in either the matrix or analyte when under the vacuum conditions of the mass spectrometer. MS/MS analyses of the low intensity peaks were performed. The dominant product ion peak of the $m / z 402$ precursor obtained at low signal-to-noise was $m / z 384$, corresponding to water loss from $\mathrm{m} / \mathrm{z}$ 402. This finding provides no support for the formation of hydroperoxides as the main products of oxidation. MS/MS data of the $m / z 400$ precursor produced similar spectra due to the "Timed Ion Selector" of the ABI 4800 being unable to effectively separate the $\mathrm{m} / \mathrm{z} 400$ and 402 precursor ions.

To explain the presence of radical cation peaks, a new mechanistic pathway involving reaction of neutral cholesterol with available radicals has been developed. Although Bourcier et al. [25] considered the possibility that $\mathrm{OH}^{*}$ could be produced during MALDI irradiation via decompositions of $\mathrm{DHB}^{+}$, based upon follow-up EI experiments, they ultimately discounted this pathway and instead attributed 

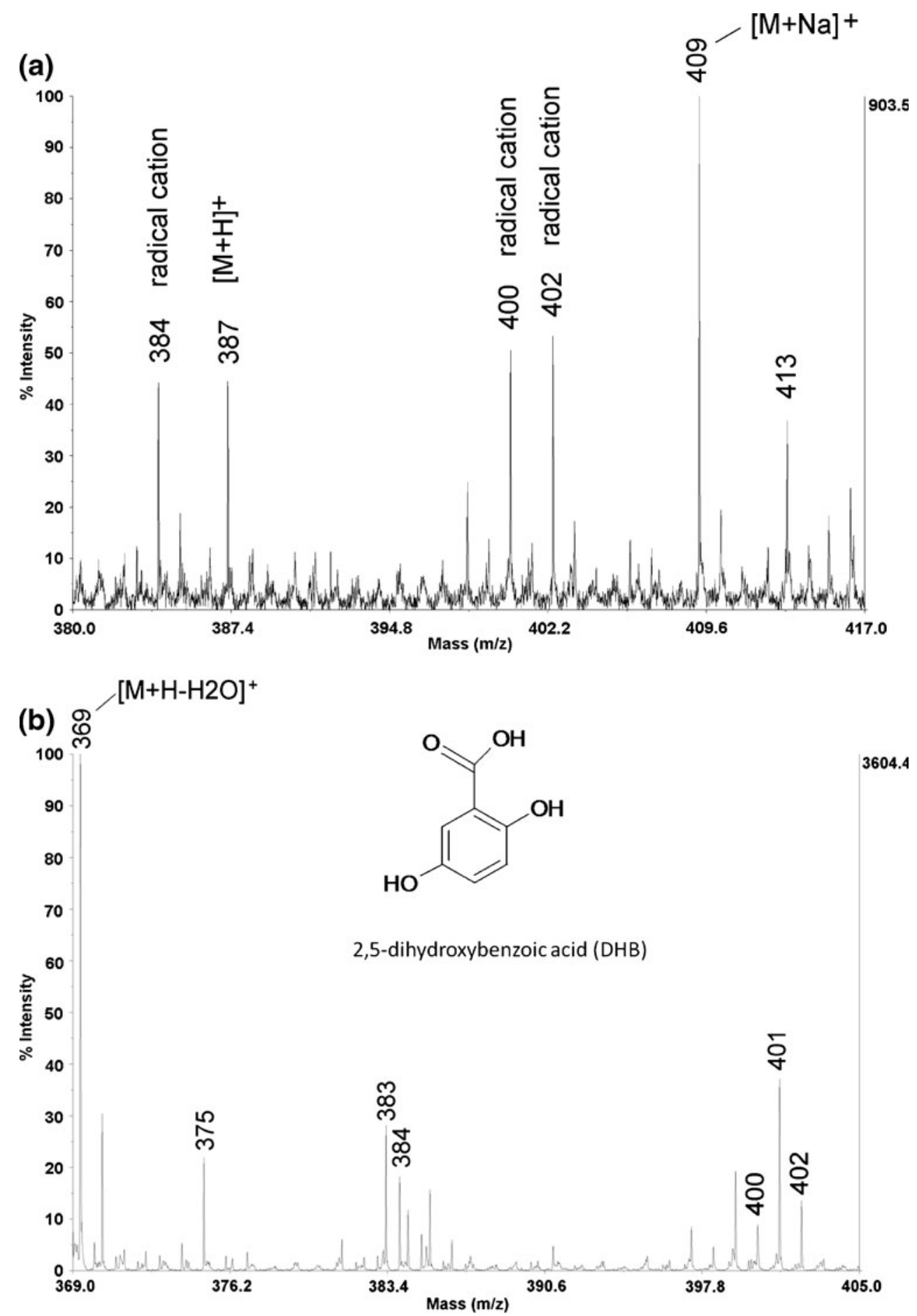

Figure 1. MALDI-TOF mass spectra of cholesterol prepared in: (a) $\mathrm{CHCl}_{3}: \mathrm{CH}_{3} \mathrm{OH}$ solution acquired at a laser intensity of 4150 with DHB as the matrix. Average of 400 shots; (b) acetonitrile with DHB as the matrix acquired at a laser intensity of 4150

the matrix peak at $m / z 137$ to $\left[\mathrm{DHB}+\mathrm{H}-\mathrm{H}_{2} \mathrm{O}\right]^{+}$rather than $\left[\mathrm{DHB}^{+\cdot}-\mathrm{OH}^{+}\right.$. In our proposed mechanism, laser irradiation of the DHB matrix yields $\mathrm{OH}^{-}$that can attack the neutral cholesterol molecule. The initial product after attack by $\mathrm{OH}^{\prime}$ is the neutral species with a molecular weight of $403 \mathrm{Da}$ (Scheme 2). Protonation of this neutral will give a peak at $m / z 404$ (not shown in scheme). This peak is not detected frequently, hence, it is likely that it and/or its
403 Da neutral precursor are highly unstable and undergo rapid decomposition. In losing molecular hydrogen from the 403 Da neutral, another neutral species of molecular weight $401 \mathrm{Da}$ is formed, which is believed to be a thermodynamically favored product that is stabilized via resonance (Scheme 2). If this free radical were to become protonated at the hydroxyl group, it could readily lose water to form $\mathrm{m} / \mathrm{z}$ 384. If protonation of the $401 \mathrm{Da}$ neutral occurred at the 
carbonyl site a relatively stable $\mathrm{m} / \mathrm{z} 402$ would result. Alternatively, the $401 \mathrm{Da}$ species can also lose another $\mathrm{H}_{2}$, forming the neutral $399 \mathrm{Da}$ species. Protonation of the 399 Da species is most likely to occur at the carbonyl group thereby giving $\mathrm{m} / \mathrm{z} 400$. If instead, protonation were to occur at the less basic alcohol group, water loss from the $\mathrm{m} / \mathrm{z} 400$ species giving $\mathrm{m} / \mathrm{z} 382$ would be expected, but was not observed.

\section{Concentration Influence}

Because cholesterol has a rather poor ion yield, higher initial solution concentrations (near $100 \mu \mathrm{M}$ ) were originally used in the sample preparation in the hopes of boosting signal strengths. However, it was noticed that when the initial solution concentration was decreased, the appearance of the radical cation peaks became more frequent. At an initial

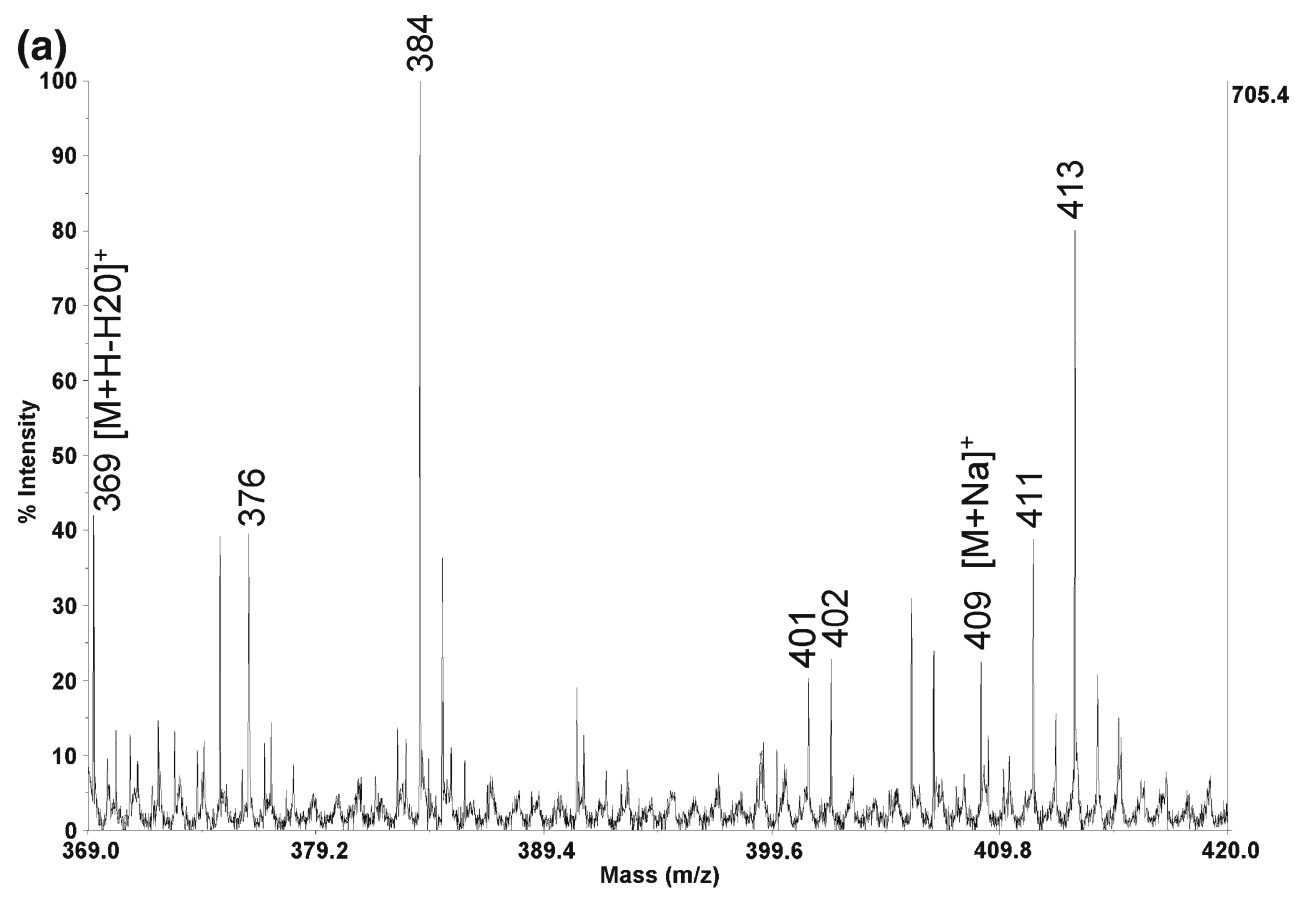

(b)

志

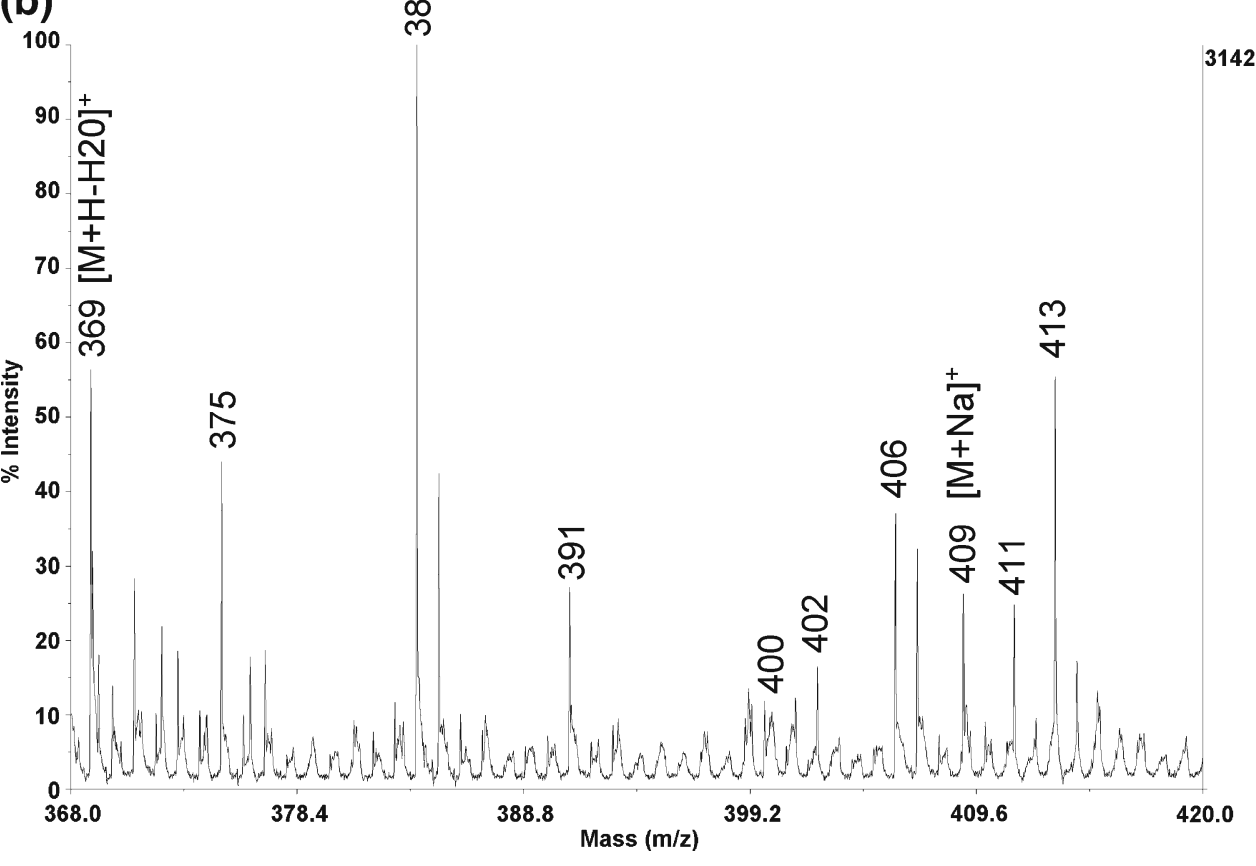

Figure 2. (continued) 

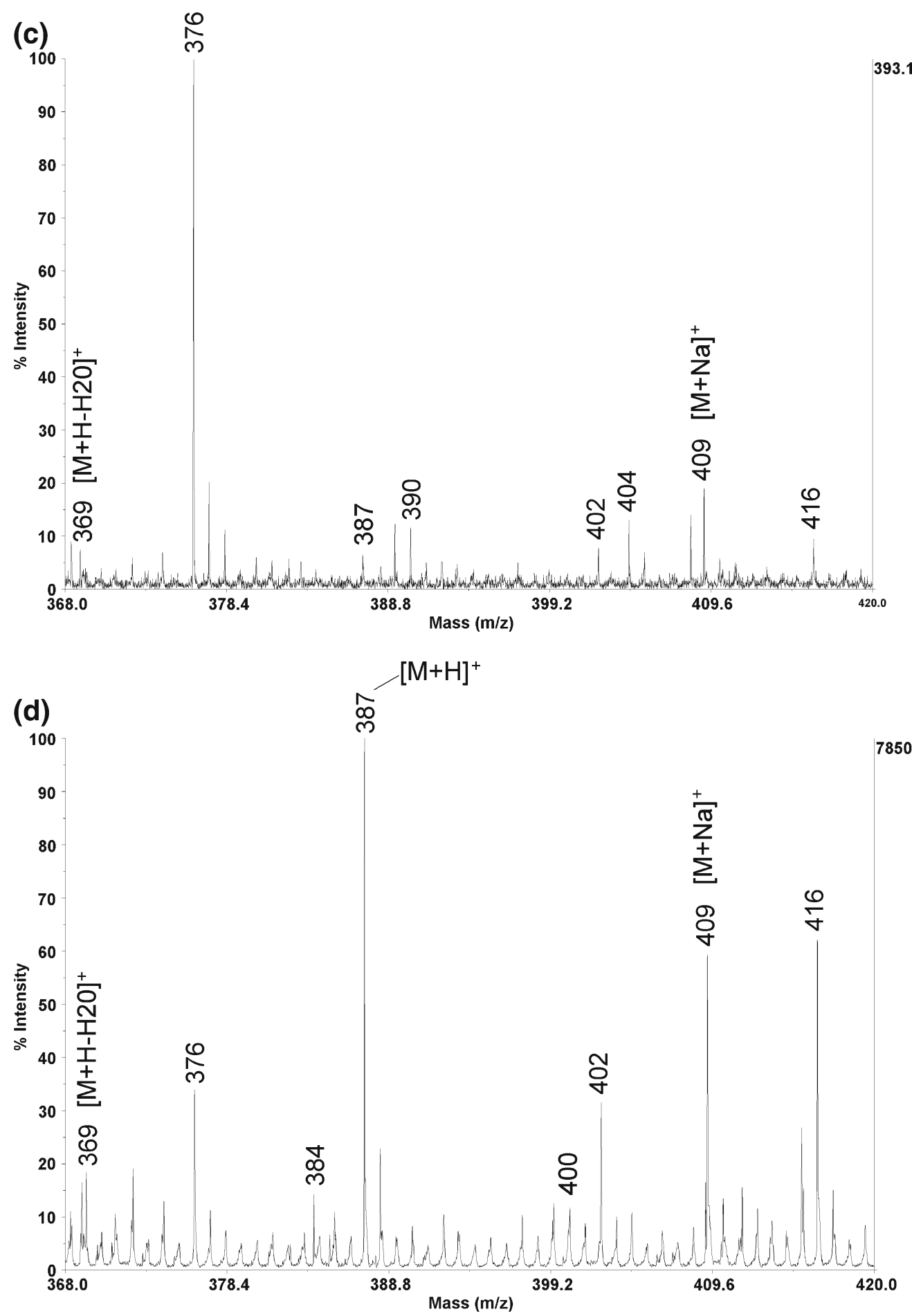

Figure 2. MALDI-TOF mass spectra of cholesterol prepared in (a) 10:1 $\mathrm{CH}_{3} \mathrm{OH}: \mathrm{H}_{2} \mathrm{O}$ acquired at a laser intensity of 4150; (b) $10: 1 \mathrm{CH}_{3} \mathrm{OH}: \mathrm{H}_{2} \mathrm{O}$ acquired at a laser intensity of 5050 ; (c) $1: 1 \mathrm{CHCl}_{3}: \mathrm{CH}_{3} \mathrm{OH}$ acquired at a laser intensity of 4150 ; and (d) $1: 1$ $\mathrm{CHCl}_{3}: \mathrm{CH}_{3} \mathrm{OH}$ acquired at a laser intensity of 5050 . DHB was used as the matrix for all samples

solution concentration of $1 \mu \mathrm{M}$ in $\mathrm{CHCl}_{3}: \mathrm{CH}_{3} \mathrm{OH}$ using DHB as the matrix, the presence of all three radical cation peaks appeared on a regular basis. Figure 3 shows a graph of normalized radical cation peak intensity vs. initial solution concentration in $\mathrm{CHCl}_{3}: \mathrm{CH}_{3} \mathrm{OH}$. The peak intensity was normalized by taking the absolute intensity of the listed peak and dividing by the intensity of a known DHB peak $(\mathrm{m} / \mathrm{z}$ 273 ) in the spectrum. The amount of DHB present remained constant as the amount of cholesterol was varied. All spectra were acquired at a laser intensity of 4150 . Each point constitutes an average of 10 spots; at each spot, 2500 laser shots were averaged. 


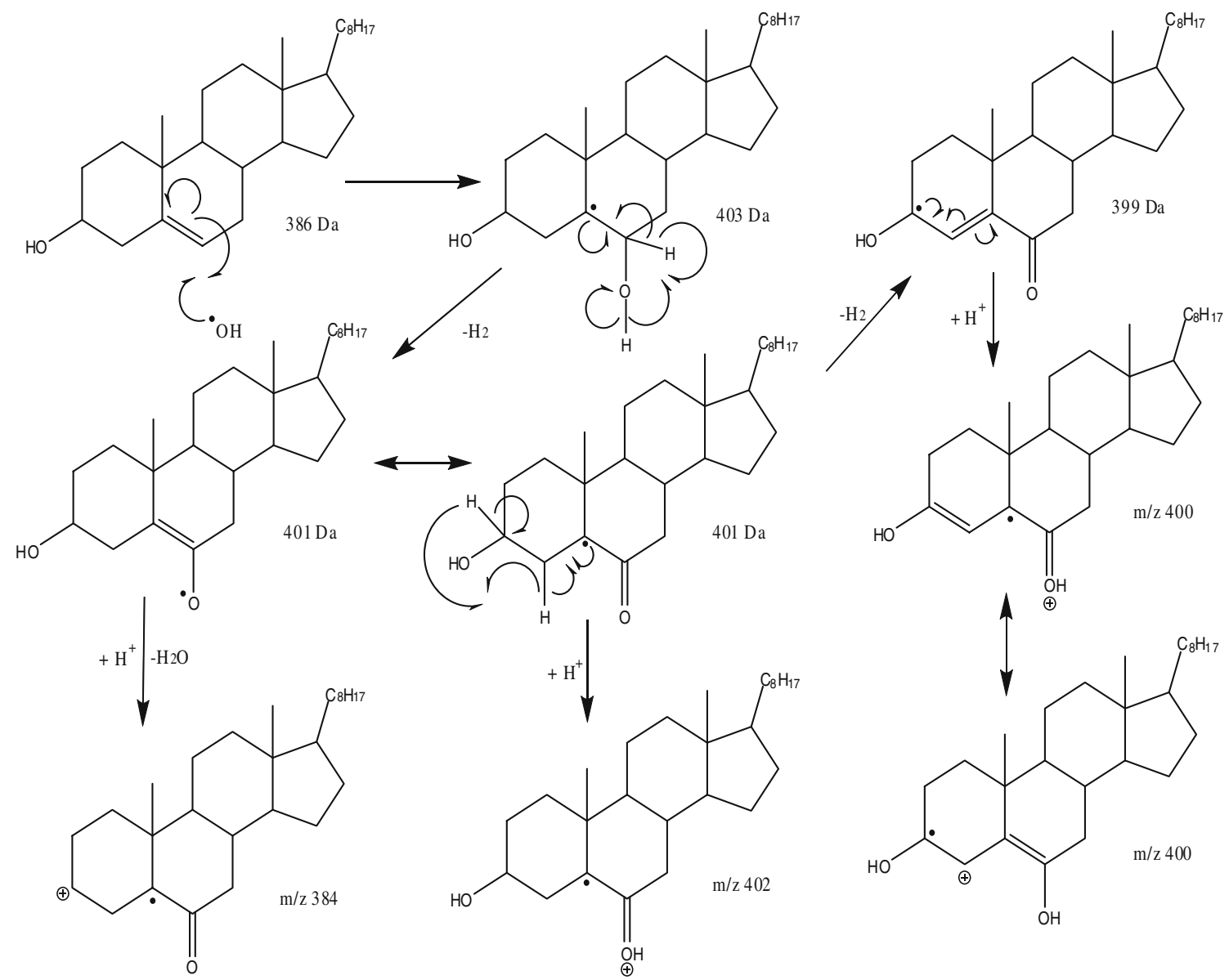

Scheme 2. Proposed mechanism of cholesterol oxidation involving initial hydroxyl radical attack on neutral cholesterol following laser irradiation during MALDI

Starting at the high end of the cholesterol concentration scale in the Figure 3 plot, it can be seen that at the highest point of initial cholesterol concentration, the signals for the three radical cation peaks and protonated cholesterol have decreased relative to the signals obtained at lower concentration. We attribute this downturn in signal at the highest cholesterol concentration to a diminished UV absorbance, hence, decreased energy deposition owing to the lower density of matrix molecules. If indeed hydroxyl radical formation from DHB is diminished at the highest cholesterol concentration (where DHB density is lowest), then it is consistent with our proposed mechanism (Scheme 2) to observe a reduction in signal intensities for $\mathrm{m} / \mathrm{z} 384,400$, and 402. Similarly, a diminished absorbance level may disfavor proton transfer reactions from the DHB matrix, which can account for the decrease in $\mathrm{MH}^{+}$formation.

At the lowest concentration of cholesterol, we propose that hydroxyl radical production from DHB is highest, due to an improved absorbance when the matrix density is at a maximum. However, there are few cholesterol molecules with which to react, hence, the signals for $m / z 384,400$, and 402 are weak. Proton transfer from the matrix is likely quite favorable in this lowest concentration regime, but there are few cholesterol molecules to accept protons, resulting in a low $\mathrm{MH}^{+}$signal. The two intermediate concentrations in Figure 3 represent situations where there is a better balance between cholesterol abundance and hydroxyl radical or proton availability, thus higher signals were observed.

\section{COP Induction and Inhibition}

Previous studies have attempted to regulate the formation of cholesterol oxidation products by means of radical inducers and radical scavengers $[3,9,26,28]$. These studies have mainly been done in solution. We have used the traditional free radical scavengers $\alpha$-tocopherol $[27,28]$ and tertiarybutyl hydroquinone [26] in an attempt to diminish the intensity of the radical cation peaks in the gas phase. The addition of TBHQ was ineffective at lowering the radical cation peaks of cholesterol dissolved in acetonitrile. However, TBHQ was effective at decreasing the presence of the $\mathrm{m} / \mathrm{z} 400$ and 402 peaks from solutions prepared in $\mathrm{CH}_{3} \mathrm{OH}$ : $\mathrm{H}_{2} \mathrm{O}$ and solutions prepared in $\mathrm{CH}_{3} \mathrm{OH}: \mathrm{CHCl}_{3}$. $\alpha$-tocopherol was only able to suppress the $\mathrm{m} / \mathrm{z} 400$ and 402 peaks in solutions prepared in $\mathrm{CH}_{3} \mathrm{OH}: \mathrm{H}_{2} \mathrm{O}$. Both radical scavengers were able to eliminate the peak at $m / z 401$, which will be discussed later. Neither of the free radical scavengers was able to eliminate the presence of the $m / z 384$ peak. 


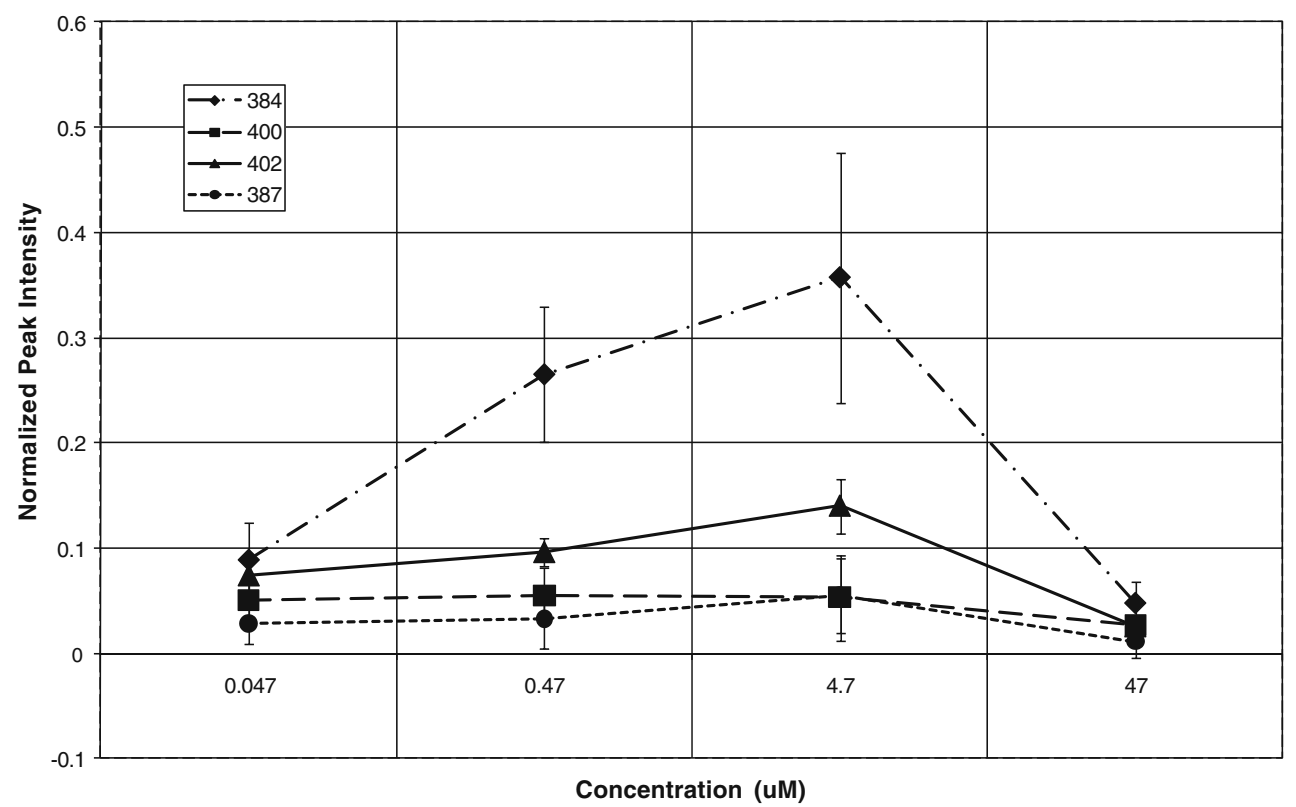

Figure 3. Normalized peak intensity vs. initial solution concentration of cholesterol from a solution of $1: 1 \mathrm{CHCl}_{3}: \mathrm{CH}_{3} \mathrm{OH}$

In separate experiments, the radical initiator cumene hydroperoxide was added to a DHB-cholesterol solution in view of forming solution-based oxidation products of cholesterol. In blank runs, with or without matrix, cumene hydroperoxide produced no peaks at $\mathrm{m} / \mathrm{z} 384,400$, and 402 . With cholesterol on the target, addition of cumene hydroperoxide boosted the relative intensities of radical cation peaks of cholesterol species at $m / z$ 384, 400, and 402 (Figure 4) when acetonitrile was used as the solvent; comparable increases were not observed with either $\mathrm{CH}_{3} \mathrm{OH}: \mathrm{H}_{2} \mathrm{O}$ or $\mathrm{CH}_{3} \mathrm{OH}: \mathrm{CHCl}_{3}$ as solvent. Because cumene hydroperoxide is a clear source of hydroxyl radical, the fact that conditions could be found whereby an increase in the intensities of the three radical cation peaks was observed in the presence of cumene hydroperoxide serves to corroborate our postulated mechanism of initial hydroxyl radical attack on neutral cholesterol (Scheme 2).

In the examples above, the fact that the choice of solvent played a critical role in determining whether the

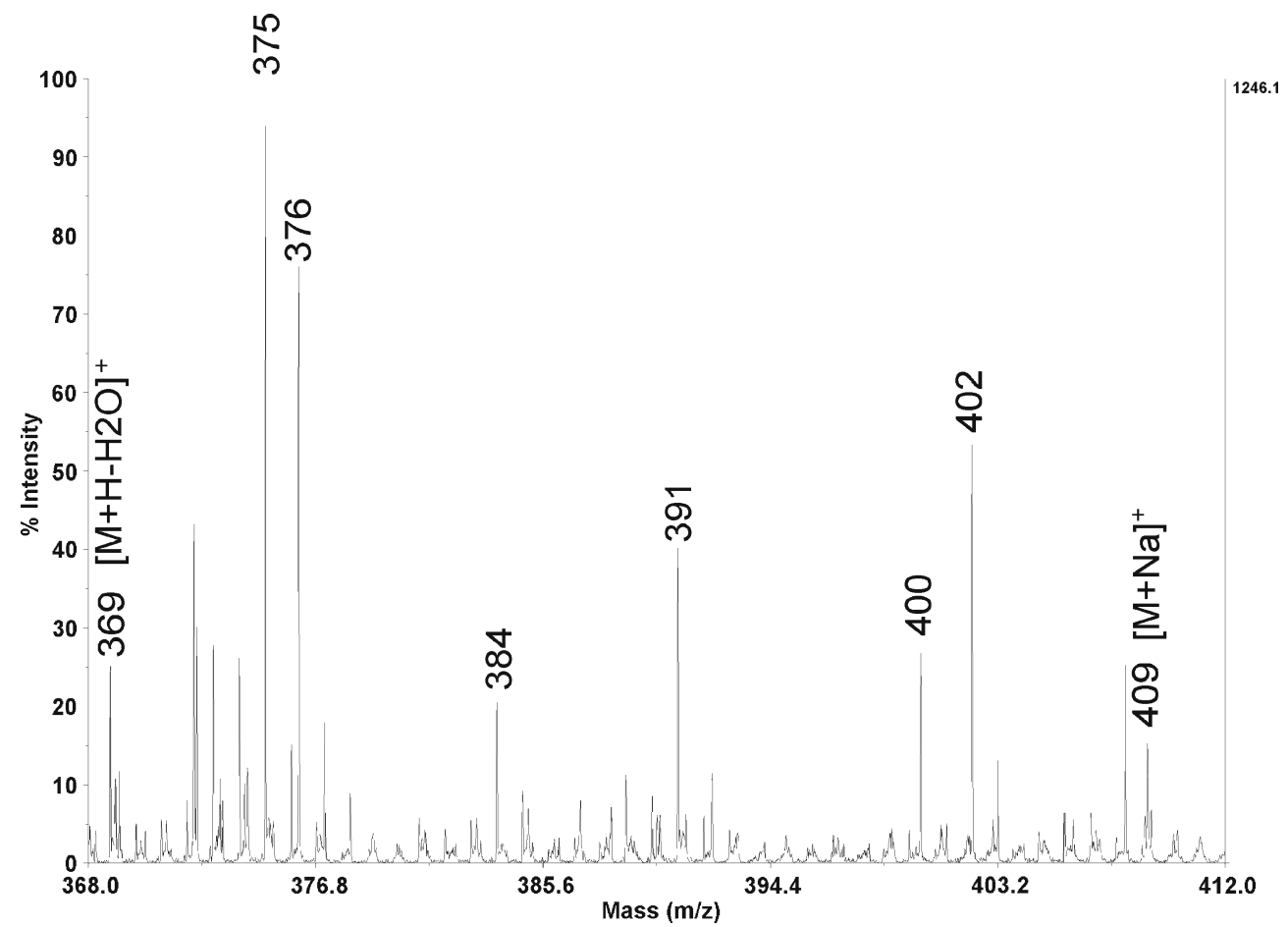

Figure 4. MALDI-TOF mass spectrum of cholesterol dissolved in acetonitrile with the addition of cumene hydroperoxide. DHB was used as the matrix 
addition of radical scavengers was able to suppress, or the presence of radical initiators was able to promote, the formation of radical species suggests that cocrystallization phenomena are exerting influences on the outcome. The solvent may influence the proximity of cholesterol and additive molecules in matrix crystals and thus affect the interaction between hydroxyl radical and cholesterol during ablation and during the earliest phase of plume expansion, prior to these species entering the gas phase.

\section{Solution Based Oxidation}

Cholesterol is known to readily oxidize in solution in the presence of air, light, free radical initiators, and/or elevated temperatures [29]; thus, it is possible that cholesterol may have been oxidized prior to analysis during storage. To test whether radicals may have been formed by pathways that do not involve laser irradiation, COP determinations were performed in solution by ES-MS. Figure 5a shows cholesterol in acetonitrile at a concentration of $155 \mu \mathrm{M}$. Figure $5 \mathrm{~b}$
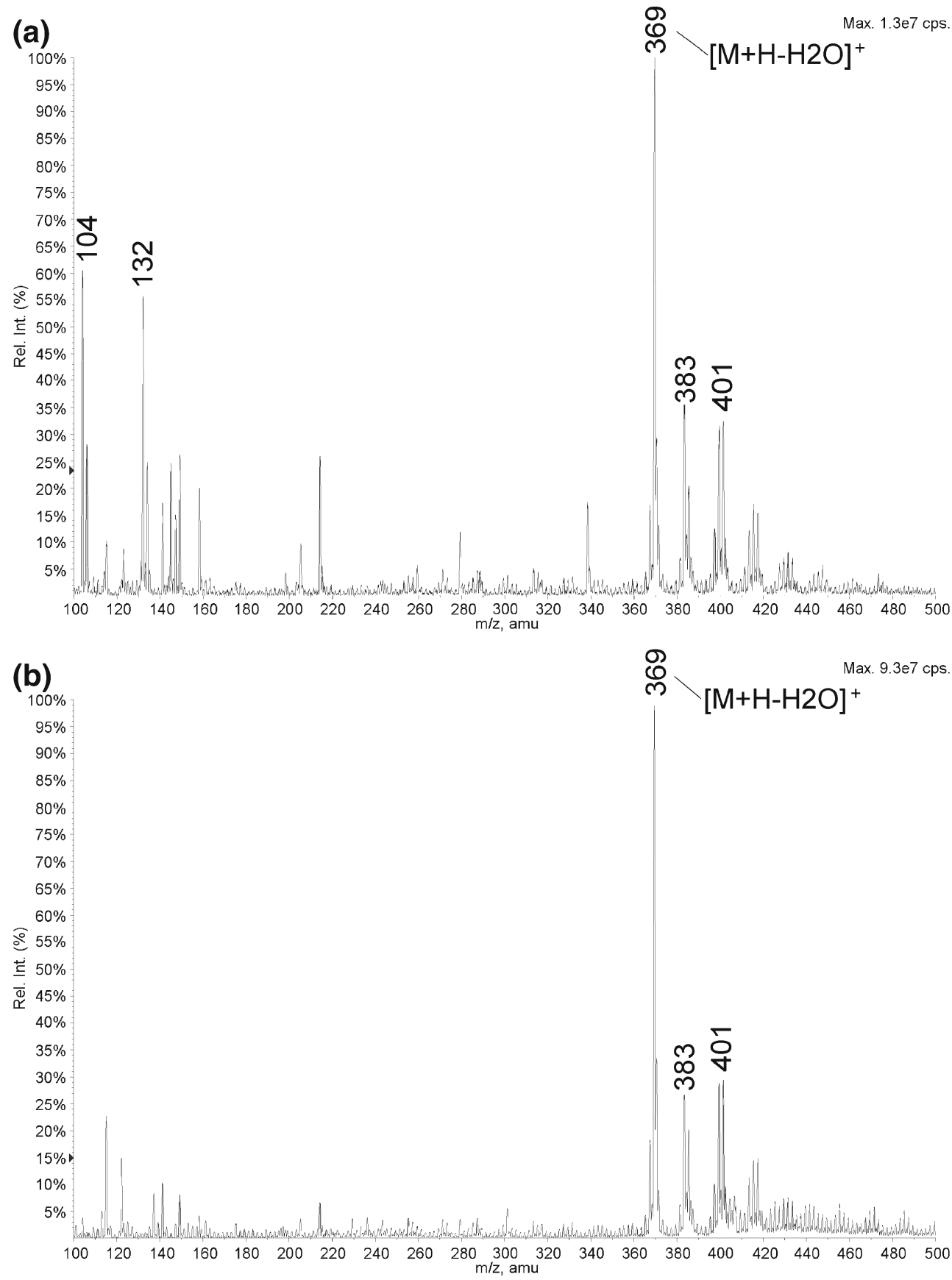

Figure 5. Electrospray mass spectra of cholesterol in (a) acetonitrile at a concentration of $155 \mu \mathrm{M}$; (b) $\mathrm{CH}_{3} \mathrm{OH}$ at a concentration of $725 \mu \mathrm{M}$ 
shows cholesterol in pure $\mathrm{CH}_{3} \mathrm{OH}$ at a concentration of $725 \mu \mathrm{M}$. The peak at $m / z 401$ has been reported in previous literature as protonated 5-cholesten-3 $\beta$-ol-7-one (Figure 5a, structure of neutral in Scheme 1f), a well known COP [11,30]. This suggests that the peak at $\mathrm{m} / \mathrm{z} 401$ in the MALDI mass spectra represents a solution-based oxidation product. The radical cation peaks at $\mathrm{m} / \mathrm{z} 384,400$, and 402 , however, cannot be detected by ES-MS, thus corroborating the assertion that the latter peaks are artifacts of the MALDI process.

\section{Comparison to Known Oxidation Products}

Recent work by Van der Brink et al. [31] has provided another possible mechanism for formation of radical oxidation products of cholesterol. Using MALDI-FTICR, Van der Brink et al. [31] claim that the peak at $\mathrm{m} / \mathrm{z} 384$ originates from either 5-cholesten-3-one $\left(\mathrm{M}^{+}\right)$, 5-cholesten3,7-diol $\left(\mathrm{M}^{+}-18\right)$, or cholest-5,6-epoxide-3-ol $\left(\mathrm{M}^{+}-18\right)$. The peak at $\mathrm{m} / \mathrm{z} 400$ was shown to originate from 5cholesten-3-ol-7-one $\left(\mathrm{M}^{++}\right)$and the peak at $\mathrm{m} / \mathrm{z} 402$ was shown to originate from either cholest-5,6-epoxide-3-ol $\left(\mathrm{M}^{+}\right)$or 5-cholesten-3,7-diol $\left(\mathrm{M}^{-+}\right)$[31]. Van der Brink et al. [31] were also able to see a peak at $\mathrm{m} / \mathrm{z} 382$ which indicates possible protonation occurring at the hydroxyl group of the 399 Da neutral molecule in Scheme 2 (top row, right column) followed by water loss. In our laboratory, the known cholesterol oxidation products 5 -cholestene- $3 \beta, 7 \beta$-diol,

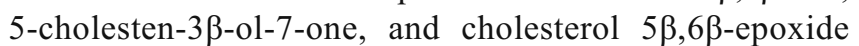
$[12,31]$ were purchased commercially and were then run on the MALDI-TOF instrument to test whether radical oxidation products would be generated from these compounds. The three known oxidation products were dissolved in $\mathrm{CHCl}_{3}: \mathrm{CH}_{3} \mathrm{OH}$ and mass spectra were acquired under conditions similar to those used for cholesterol. The MALDI-TOF mass spectra of these three oxidation products are shown in Supplemental Material. The radical cation peak at $\mathrm{m} / \mathrm{z} 384$ appeared in all three spectra, but at rather different absolute and relative intensities. The peak at $\mathrm{m} / \mathrm{z} 402$ appears only as an isotopic peak of $m / z 401$ in all three spectra. The $m / z 400$ species could not be detected in any of the standards. The latter two mass spectra (Figure b and c in Supplemental Material) exhibit sizeable $m / z 384$ peaks which may have arisen from $\mathrm{M}^{+\cdot}$ ions that had undergone rapid water loss.

\section{Conclusion}

The radical cation peaks at $\mathrm{m} / \mathrm{z} 384,400$, and 402 were deduced to be artifacts of laser irradiation during MALDI-MS of cholesterol. We propose that hydroxyl radical attack on neutral cholesterol is the first step in the formation of these open shell species; hydroxyl radical can be formed upon laser irradiation of the employed DHB matrix. Compared with DHB, the dithranol matrix produced lower intensity $\mathrm{MH}^{+}$ peaks for cholesterol, but the same radical cation peaks at $\mathrm{m} / \mathrm{z}$ 384.3430 (9.8 ppm error), 400.3326 (3.8 ppm error), and 402.3481 (4.2 ppm error) were observed. Similar to DHB, dithranol has two hydroxyl groups attached to its aromatic rings. It therefore seems reasonable that hydroxyl radicals liberated during laser irradiation could be responsible for generating the above three radical cation peaks from cholesterol in dithranol by a mechanism analogous to that shown in Scheme 2 for cholesterol in DHB. MALDI mass spectrometry must then be viewed as providing a potential means to (inadvertently) oxidize analytes during the laser irradiation process.

\section{Acknowledgments}

The authors acknowledge that financial support for this research was provided by the National Science Foundation through CHE-0518288, CHE-0611902 and DBI-0619272. Additional financial support came from the Louisiana Board of Regents through HEF(2001-06)-08.

\section{References}

1. Goldstein, J.L., Brown, M.S.: Binding and degradation of low density lipoproteins by cultured human fibroblasts. J. Biol. Chem. 249, 51535162 (1974)

2. Guyton, J.R., Black, B.L., Seidel, C.L.: Focal toxicity of oxysterols in vascular smooth muscle cell culture. Amer. J. Pathol. 137, 425-434 (1990)

3. Osada, K., Hoshina, S., Nakamura, S., Sugano, M.: Cholesterol oxidation in meat products and its regulation by supplementation of sodium nitrite and apple polyphenol before processing. J. Agric. Food Chem. 48, 3823-3829 (2000)

4. Hwang, P.L.H.: Inhibitors of protein and RNA synthesis block the cytotoxic effects of oxygenated sterols. Biochem. Biophys. Acta. 1136, 5-11 (1992)

5. Kücük, O., Stoner-Picking, J., Yachnin, S., Gordon, L.I., Williams, R. M., Lis, L.J., Westerman, L.P.: Inhibition of cytolytic T lymphocyte activity by oxysterols. Lipids 29, 657-660 (1994)

6. Ansari, G.A., Walker, R.D., Smart, V.B., Smith, L.L.: Further investigations of mutagenic cholesterol preparations. Food Chem. Toxicol. 20, 35-41 (1982)

7. Busch, T.P., King, A.J.: Artifact generation and monitoring in analysis of cholesterol oxide products. Anal. Biochem. 388, 1-14 (2009)

8. Fortier, C.A., Guan, B., Cole, R.B., Tarr, M.A.: Covalently bound fluorescent probes as reporters for hydroxyl radical penetration into liposomal membranes. Free Radic. Bio. Med. 46, 1376-1385 (2009)

9. Chien, J.T., Wang, H.C., Chen, B.H.: Kinetic model of cholesterol during heating. J. Agric. Food Chem. 46, 2572-2577 (1998)

10. Andreu, I., Bosca, F., Sanchez, L., Morera, I.M., Camps, P., Miranda, M.A.: Efficient and selective photogeneration of cholesterol-derived radicals by intramolecular hydrogen abstraction in model dyads. Org. Lett. 8, 4597-4600 (2006)

11. Uemi, M., Ronsein, G.E., Miyamoto, S., Medeiros, M.H.G., DiMascio, P.D.: Generation of cholesterol carboxyaldehyde by the reaction of singles molecular oxygen [O2 (1triangleG)] as well as ozone with cholesterol. Chem. Res. Toxicol. 22, 875-884 (2009)

12. Smith, L.L.: Cholesterol autoxidation. Plenum Press, New York (1981)

13. Wasilchuk, B.A., LeQuesne, P.W., Vouros, P.: Monitoring cholesterol auto-oxidation processes using multideuteriated cholesterol. Anal. Chem. 64, 1077-1087 (1992)

14. Stovall, J., Axelson, M.: Newer approaches to the isolation, identification, and quantitation of steroids in biological materials. Vitam. Horm. 39, 31-144 (1982)

15. Partridge, L.G., Djerassi, C.: Mass spectrometry in structural and stereochemical problems. 250. Characteristic fragmentations of cholesterol acetate. J. Org. Chem. 42, 2799-2805 (1977)

16. Sostarecz, A.G., Cannon, D.M., McQuaw, C.M., Sun, S., Ewing, A.G., Winograd, N.: Influence of molecular environment on the analysis of phospholipids by time-of-flight secondary ion mass spectrometry. Langmuir 20, 4926-4932 (2004) 
17. Tsai, L.S., Hudson, C.A.: High performance liquid chromatography of oxygenated cholesterols and related compounds. J. Am. Oil Chem. Soc. 58, 931-934 (1981)

18. Maerker, G., Nungesser, E.H., Zulak, I.M.: HPLC separation and quantitation of cholesterol oxidation products with flame ionization detection. J. Agric. Food Chem. 36, 61-63 (1988)

19. Griffiths, W.J.: Tandem mass spectrometry in the study of fatty acids, bile acids, and steroids. Mass Spectrom. Rev. 22, 81-152 (2003)

20. Wang, Y., Hornshaw, M., Alvelius, G., Bodin, K., Liu, S., Sjovall, J., Griffiths, W.J.: Matrix-assisted laser desorption/ionization high-energy collision-induced dissociation of steroids: analysis of oxysterols in rat brain. Anal. Chem. 78, 164-173 (2006)

21. Yu, H., Lopez, E., Young, S.W., Luo, J., Tian, H., Cao, P.: Quantitative analysis of free fatty acids in rat plasma using matrix-assisted laser desorption/ionization time-of-flight mass spectrometry with mesotetrakis porphyrin as matrix. Anal. Biochem. 354, 182-191 (2006)

22. Karas, M., Hillenkamp, F.: Laser desorption ionization of proteins with molecular masses exceeding 10, 000 Daltons. Anal. Chem. 60, 23012303 (1988)

23. Cohen, S.L., Chait, B.T.: Influence of matrix solution conditions on the MALDI-MS analysis of peptides and proteins. Anal. Chem. 68, 31-37 (1996)

24. Schiller, J., Zschornig, O., Petkovic, M., Muller, M., Arnold, J., Arnold, K.: Lipid analysis of human HDL and LDL by MALDI-
TOF mass spectrometry and ${ }^{31} \mathrm{P}-\mathrm{NMR}$. J. Lipid Res. 42, 1501-1508 (2001)

25. Bourcier, S., Bouchonnet, S., Hoppilliard, Y.: Ionization of 2, 5dihyrxybenzoic acid (DHB) matrix-assisted laser desorption ionization experiments and theoretical study. Int. J. Mass Spectrom. 210/211, 59$69(2001)$

26. Lai, S., Gray, J.I., Buckley, D.J., Kelly, P.M.: Influence of free radicals and other factors on formation of cholesterol oxidation products in spray-dried whole egg. J. Agric. Food Chem. 43, 1127-1131 (1995)

27. Valastyan, S., Thakur, V., Johnson, A., Kumar, K., Manor, D.: Novel transcription activities of vitamin E: inhibition of cholesterol biosynthesis. Biochemistry 47, 744-752 (2008)

28. Xu, Z., Hua, N., Godber, J.S.: Antioxidant activity of tocopherols, tocotrienols, and $\gamma$-oryzanol components from rice bran against cholesterol oxidation accelerated by 2, 2'-azobis (2-methylpropionamidine) dihydrochloride. J. Agric. Food Chem. 49, 2077-2081 (2001)

29. Maerker, G.: Cholesterol autoxidation — current status. JAOCS 64, 388 392 (1987)

30. Smith, L.L.: Review of progress in sterol oxidations: 1987-1995. Lipids 31, 453-487 (1996)

31. Van den Brink, O.F., Ferreira, E.S.B., Van der Horst, J., Boon, J.J.: A direct temperature-resolved mass spectrometry study of cholesterol oxidation products in light-aged egg tempera with examples from works of art. Int. J. Mass Spectrom. 284, 12-21 (2009) 